\title{
Dependence of Multijunction Optimal Gaps on Spectral Variability and Other Environmental and Device Parameters
}

\author{
José M. Ripalda Jerónimo Buencuerpo Iván García
}

\begin{abstract}
We present a method to calculate the yearly energy production of multijunctions including spectral variations. We use it to find the optimal band gaps yielding maximum energy production. The band gaps found are different to those previously reported when using the standard efficiency as the optimization target. Our calculations predict that novel hybrid tandems in combination with bifacial silicon can lead to energy yields near $1 \mathrm{MWh} \mathrm{m} \mathrm{mear}^{-1}$ at most locations of interest. We also discuss the effects of changing parameters such as the external radiative efficiency, series resistance, sub-cell thickness, temperatures, and location.
\end{abstract}

Index Terms-Yearly energy yield, hybrid tandems, multijunctions, bifacial.

\section{INTRODUCTION}

Using more efficient solar cells might not only result in a higher return on investment, but should also lessen the environmental and aesthetic impact of photovoltaic (PV) installations. The nominal standard efficiency of modules in utility-scale new installations is increasing by $0.6 \%$ per year [1]. At the current rate we will reach the practical limits of single junction photovoltaic technology within a decade. The only proven method to significantly increase the efficiency beyond the limits of conventional silicon technology is the use of multijunction devices, used either with or without optical concentration. But there still exists uncertainty about how the changes of the solar spectrum as a function of time affect the energy production of multijunction solar cells [2]. As reviewed by Kurtz et al., there have been many previous efforts to optimize solar cell band gaps, but in all cases the target of the optimization was aimed at maximizing the efficiency under standard conditions, and not the yearly energy production [3], [4].

In a recently published paper, [5] we show that data sets with thousands of solar spectra can be clustered to a few characteristic proxy spectra, and use these proxy spectra to find the band gaps that maximize the yearly energy production. We find that previously reported multijunction band gaps resulting in maximum efficiency in standard conditions are generally not the ones that maximize the energy production. Here we use our methodology to study the effects of changing various parameters such as the external radiative efficiency, series resistance, sub-cell thickness, temperatures, and location. We also evaluate hybrid multijunction systems in combination with bifacial silicon.

\section{TANDEM ENERGY PRODUCTION}

We have used a modified detailed balance method with a set of proxy spectra obtained from clustering of yearly spectral sets as described in Ref. [5]. The yearly spectral set used here and in our previous work was obtained from the SMARTS 2.9 .5 code at a fixed latitude of $40^{\circ} \mathrm{N}$ [6]. Using as a reference the National Solar Radiation Database (NSRDB) data for Boulder, Colorado [7], we obtain empirical corrective factors for the energy yield to include the effect of clouds and other atmospheric phenomena not accounted for by SMARTS 2.9.5. Full details of the methodology can be found in Ref. [5].

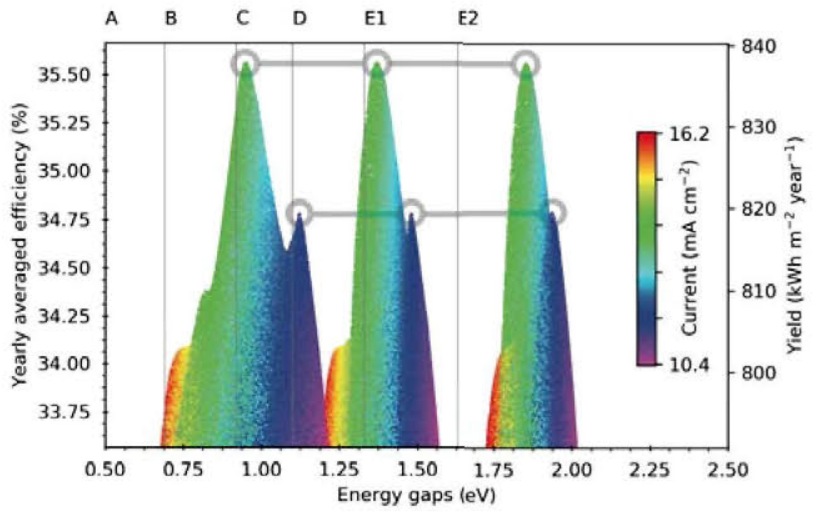

Fig. 1. Energy production for triple junction current matched devices with two axis tracking but without concentration. Band gaps are randomly chosen. Two sets of near optimal band gaps are highlighted to illustrate that points with the same efficiency value and the same color (average photocurrent) are junctions belonging in the same tandem device. Labels on top correspond to the low energy thresholds of each of the atmospheric transmission bands using the nomenclature defined by McMahon [4]. All figures below share the same color scale.

We calculate the energy production for randomly generated combinations of band gaps to find all local maxima. An example corresponding to triple junctions without concentration is shown in Fig. 1. These plots can be used to find out how to adjust the band gaps to minimize the efficiency loss when the optimal band gap combination is not attainable, and to quantify such efficiency loss. Several local minima can be found within $2 \%$ of the global maximum. The band gap of the bottom junction largely determines the short circuit current 
and the optimal band gaps for the other junctions. Bottom junctions placed at the low energy edge of each of the atmospheric transmission bands result in different local efficiency maxima as a function of the band gaps. A more detailed analysis of the effect of atmospheric transmission bands on optimal band gaps is offered by McMahon et al. [4].

\section{PARAMETER SENSITIVITY ANALYSIS}

Because we generate random combinations of band gaps, most combinations are sub-optimal due to spectral and current mismatch. For further analysis we have discarded most band gap combinations in Fig. 1 and retained only those within $0.1 \%$ of the maximum efficiency at each current level. The remaining data is represented in Fig. 2 as a function of the short circuit current of each device. The current serves as a single parameter to characterize the average band gap energy. The band gaps corresponding to each data point can be obtained by comparison with Fig. 1 as the color scale is the same.

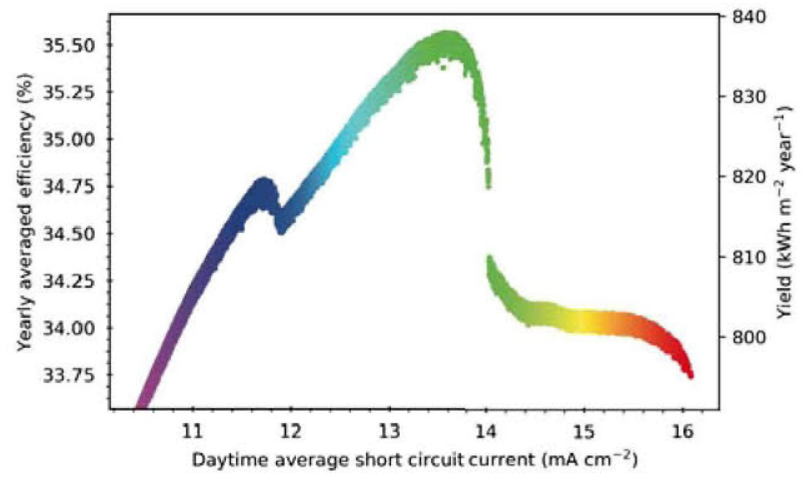

Fig. 2. Energy yield as a function of the yearly averaged short circuit current for a random set of near optimal triple junctions. The color scale is that in Fig. 1

Using the same band gap combinations and the same horizontal axis as in Fig. 2, we present in Fig. 3 the energy yield change resulting from changing the latitude from $40^{\circ}$ to $60^{\circ}$. Atmospheric conditions remain unchanged. The band gaps can be identified by correspondence with the color scale in Fig. 1. The yield reduction is almost the same for all devices and is mostly given by the reduced average irradiance. Similar results (not shown) are found when changing the latitude from 0 to $40^{\circ}$. The spikes at 120,140 , and 160 $\mathrm{A} \cdot \mathrm{m}^{-2}$ are due to the bottom junction band gap crossing an absorption band threshold. As devices at such thresholds are not optimal, the relevant trend is that shown by devices away from the spikes. The data in Fig. 3 suggests that tandems built with high band gap materials are slightly more sensitive to a change in location latitude.

We have also studied the effect of changing a number of device and material properties. The most obviously predictable effect is that of the series resistance in Fig. 4, with resistance losses increasing monotonically with the current as the resistance is doubled from $0.4 \Omega \mathrm{cm}^{2}$ to $0.8 \Omega \mathrm{cm}^{2}$.

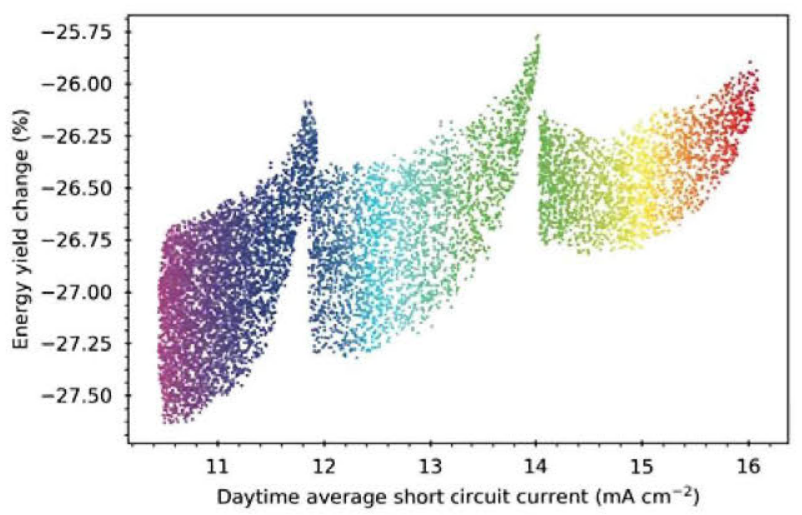

Fig. 3. Energy yield change resulting from increasing the latitude from $40^{\circ}$ to $60^{\circ}$. The yearly averaged efficiency of the best device goes up very slightly from $35.56 \%$ to $35.58 \%$

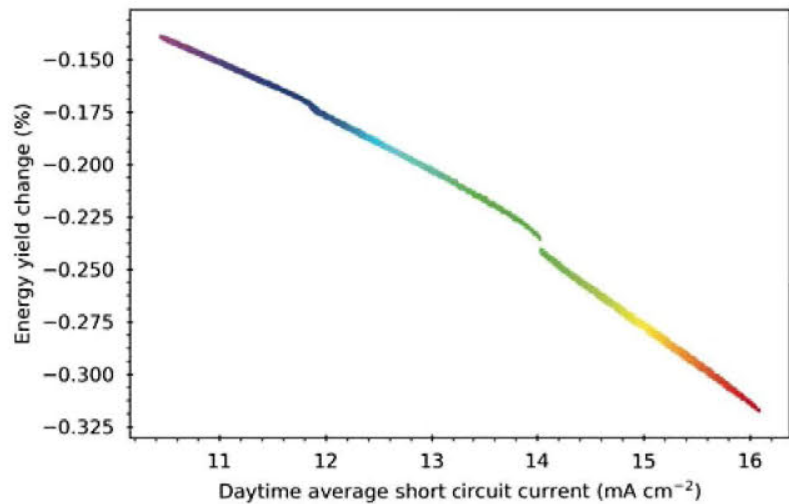

Fig. 4. Energy yield change resulting from doubling the series resistance. The yearly averaged efficiency of the best device goes down from $35.56 \%$ to $35.48 \%$

The quality of the back-side mirror affects the probability of photon recycling, and consequently the open circuit voltage, resulting in a slight increase of the energy yield (Fig. 5). This energy yield increase is slightly larger for tandems based on low band gap materials with high short circuit currents. This follows from the Schockley-Queisser detailed balance theory, because electroluminescence losses affect the voltage independently of the band gap.

As seen in Fig. 6, the larger effect observed is that of the external radiative efficiency, highlighting the difficulty of obtaining high energy yields with materials other than III$\mathrm{V}$ semiconductors. Although radiative efficiencies as high as $20 \%$ are attainable with a few binary compounds such as GaAs, the values chosen for the ERE, $0.1 \%$ and $1 \%$, are representative of most high quality III-V ternary alloys, with and without threading dislocations due to metamorphic epitaxy, respectively. Our results therefore suggest that if the use of metamorphic, or otherwise defective, materials is required, it is preferable to use high band gap materials.

We also find (not shown) that when increasing the spectrally integrated transmission of the top and middle sub- 


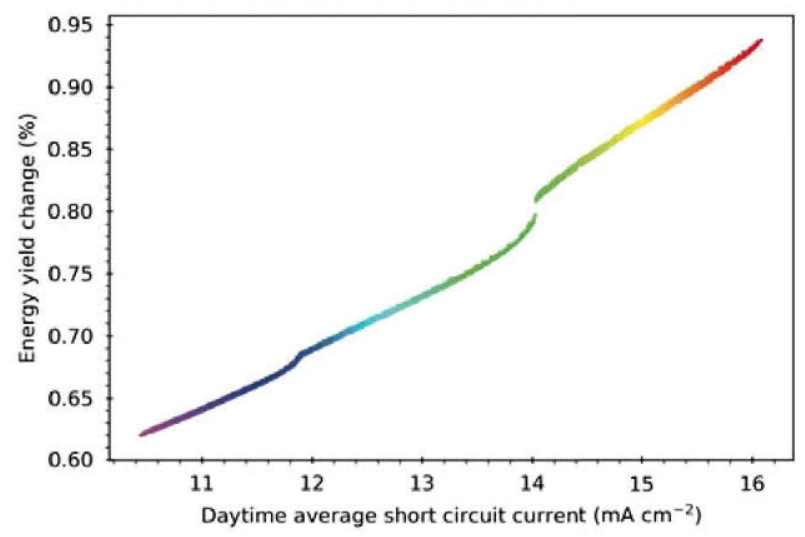

Fig. 5. Energy yield change resulting from replacing the backside semiconductor/air interface with a semiconductor/metal interface with perfect reflectivity. The yearly averaged efficiency of the best device goes up from $35.56 \%$ to $35.83 \%$

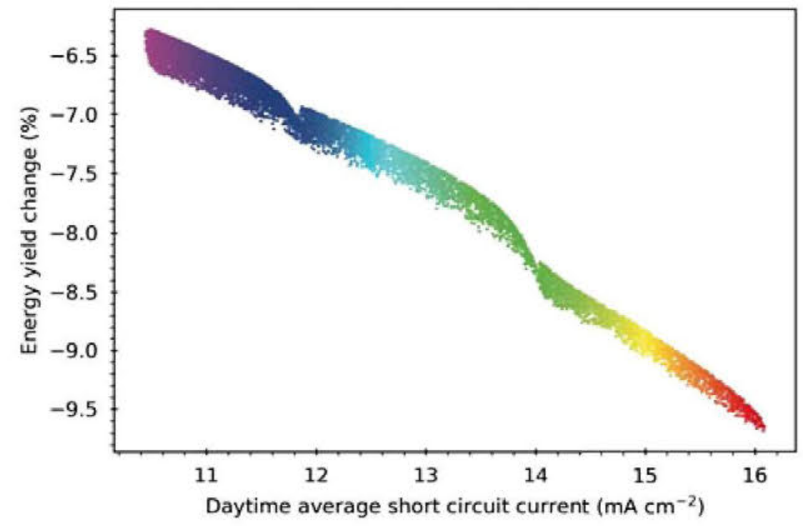

Fig. 6. Energy yield change resulting from decreasing the external radiative efficiency from $1 \%$ to $0.1 \%$. The yearly averaged efficiency of the best device goes down from $35.56 \%$ to $32.79 \%$

cells from $2 \%$ to $10 \%$ (by decreasing the thickness), it is possible to readjust the band gaps so that the efficiency drop is $1 \%$ (3\% drop in energy yield). Without readjusting the band gaps, the drop is from $35.56 \%$ to $33.86 \%$. This is relevant in those cases where sub-cell thickness is limited by the carrier diffusion lengths, accumulated lattice mismatch stress, cost and sustainability considerations, or other material constraints.

\section{SPECTRAL AND TEMPERATURE VARIABILITY}

To study the effect of atmospheric conditions we have used global horizontal spectral irradiance data from the National Solar Resource DataBase at two locations (Reno and Philadelphia) with the same latitude $\left(40^{\circ}\right)$ but very different atmospheric conditions [7]. The result (Fig. 7) is given mostly by the reduced irradiance at the cloudiest location, and depends only very slightly on the band gaps. This result suggests that, except perhaps in extreme cases [8], multijunctions should not be much more sensitive to the deployment location than conventional single junction technology, and in both cases the uncertainties on the predicted yearly energy yield are mostly given by the large uncertainties in the spectrally integrated irradiance.

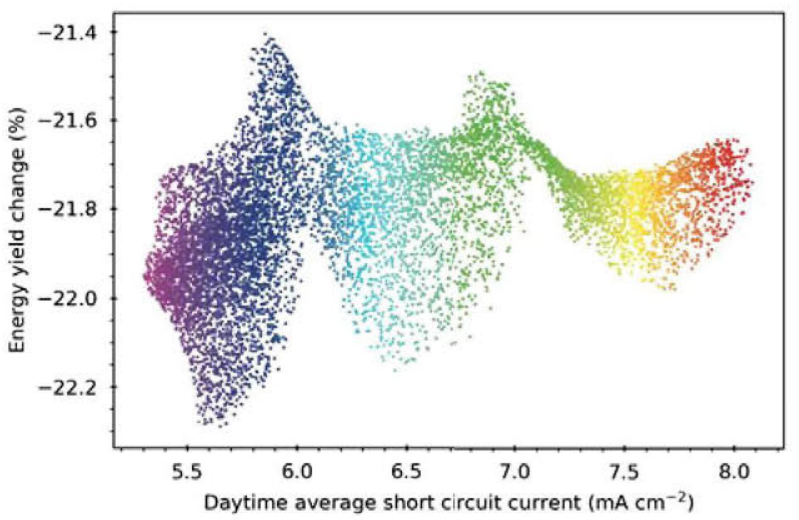

Fig. 7. Energy yield change as the location changes from Reno to Philadelphia.

We have studied the effect of the yearly variability of the temperature and the solar spectrum; separately, and their combined effect. The yearly effect of spectral variations on the optimal 1-sun triple junction is an absolute drop of $0.78 \%$ for the average efficiency. As seen in Fig. 8, devices with high band gap energies are most sensitive to spectral variations. On the contrary, these are the least sensitive to the effects of temperature variations (Fig. 9). The yearly effect of temperature variations on the optimal 1-sun triple junction is an absolute drop of $0.96 \%$ for the average efficiency, slightly larger than the effect of spectral variations.

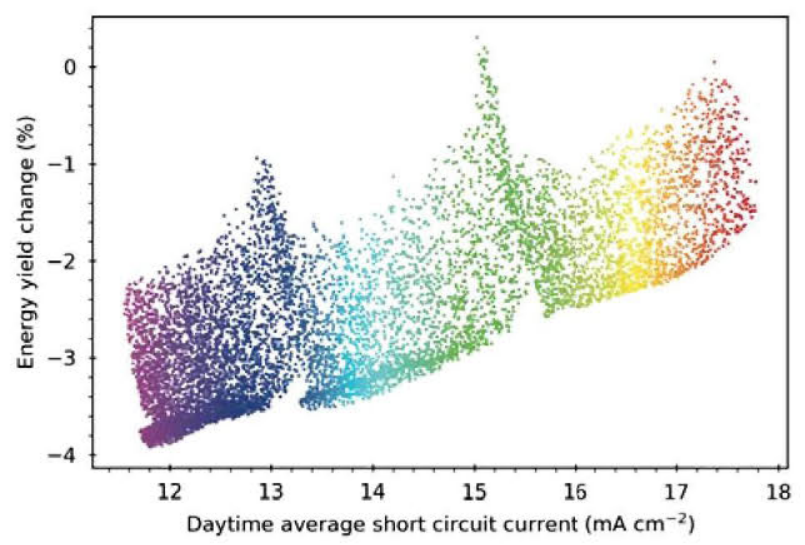

Fig. 8. Energy yield change resulting from yearly spectral variability in comparison with the energy yield obtained from the ASTM G173-03 standard spectrum. The yearly averaged efficiency of the best device goes down from $36.34 \%$ to $35.56 \%$

Even though both the effects of temperature and spectral variations are markedly different depending on the short circuit current, surprisingly the trends in each of these two effects compensate so that the combined effect of spectral and temperature variations is nearly the same for all near optimal 


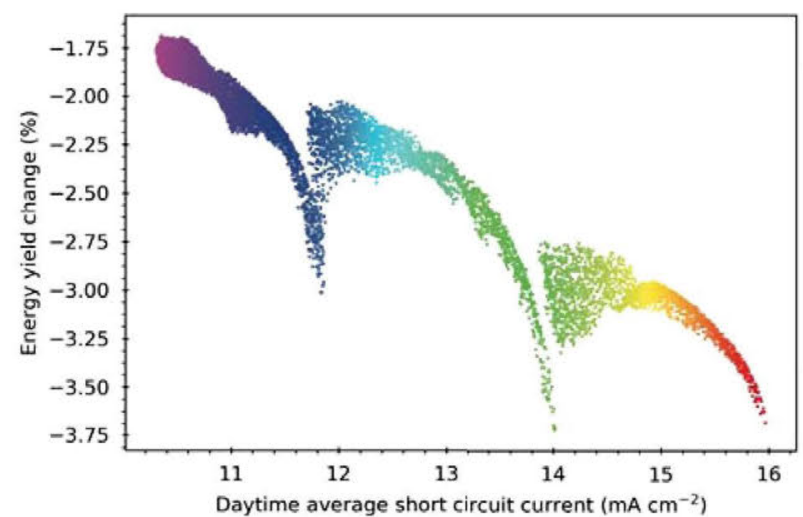

Fig. 9. Energy yield change resulting from yearly temperature variability in comparison with the energy yield obtained at a fixed temperature of $300 \mathrm{~K}$. The yearly averaged efficiency of the best device goes down from $36.52 \%$ to $35.56 \%$

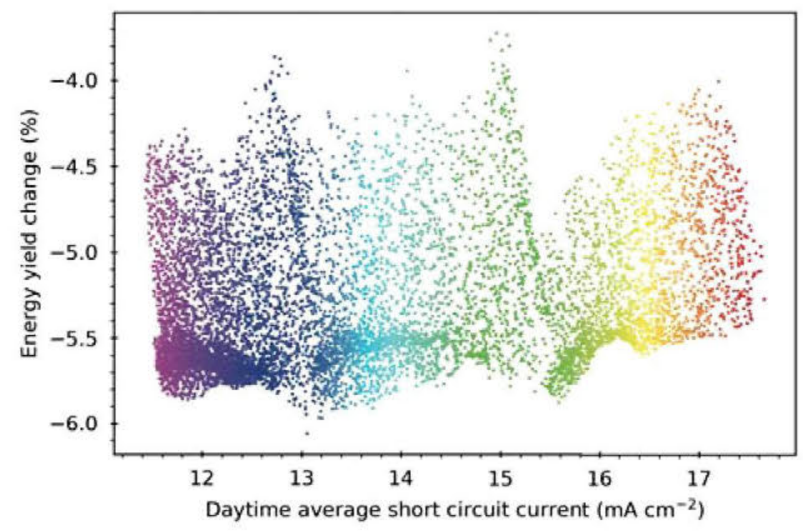

Fig. 10. Energy yield change resulting from the combined effects of yearly spectral and temperature variability in comparison with the energy yield obtained in standard conditions. The yearly averaged efficiency of the best device goes down from $37.53 \%$ to $35.56 \%$.

band gap combinations, as illustrated in Fig. 10. This coincidental cancellation of both trends is unlikely to be complete in all locations. The optimal 3 junction flat plate device has an average performance degradation of $1.97 \%$ in terms of absolute efficiency, revealing that the effects of temperature and spectral variability are not linearly additive. Near optimal two terminal, 6 junction concentrator devices (not shown) follow very similar trends, with a $3.16 \%$ efficiency drop from $50.19 \%$ to $47.03 \%$.

\section{HYBRID MODULES}

Stacking a multijunction device on top of bifacial silicon leads to high energy production [5], [9]. In this section we use the methodology we developed in Ref. [5] to evaluate three types of hybrid modules combining multijunctions and bifacial silicon. We only consider here the case where the bifacial silicon is electrically independent of the other components, because two terminal monolithic integration still faces a number of challenges, and the energy yield of 3 and 4 terminal tandems is slightly higher [5].

\section{A. Flat Plate Hybrids}

Mechanically stacked tandems built on top of bifacial silicon operating without optical concentration will be here called flat hybrids to distinguish them from hybrids based on concentrator technology. III-V semiconductors have demonstrated excellent 1-sun performance on top of silicon [9], but using III-V materials without optical concentration faces complex challenges in terms of sustainability and cost [10]. Perovskites have demonstrated silicon based double junctions with efficiency beyond the limits of silicon technology [11], but these devices, so far, are unstable and unreliable compared with silicon or III-V devices.

\section{B. Concentrator Soldered Hybrids}

At present, multijunctions based on III-V materials are the only ones that have demonstrated both long term stability and high efficiency [12]. Even though the cost of these materials per unit area seems to be an order of magnitude too high to compete with silicon in 1-sun flat plate applications, the required solar cell area can be reduced by 3 orders of magnitude by using optical concentrators and two axis tracking (concentrator photovoltaics, CPV). One of the shortcomings of conventional CPV is that diffuse light is wasted. To address this issue, several authors have proposed covering the whole back plate of the concentrator module with silicon solar cells. This idea dates back at least to a 1993 patent application [13]. More recently Benitez et al. have proposed multijunction concentrator modules with bifacial silicon solar cells to harvest not only the diffuse light, but also the albedo irradiance. Due to the high thermal conductivity of silicon, these bifacial cells serve the dual purpose of acting as thermal spreaders for the III-V solar cells mounted on top [14], [15]. Additionally, some of the direct irradiance is scattered by imperfections in the concentrator optics and is collected by the silicon on the back of the module. These hybrid modules with small concentrator solar cells stacked on top of silicon solar cells are already being tested experimentally by several research groups [15]-[18]. The silicon substrate can also be used as a printed circuit board for III-V cell interconnection, thus this type of module will here be called soldered hybrids.

\section{Concentrator Optically Coupled Hybrids}

The added value resulting from including bifacial silicon in a concentrator module can be increased if silicon harvests not only the diffuse and albedo irradiance, but also part of the direct irradiance in the appropriate spectral band. Mohedano et al. proposed a variant of this idea where a dichroic mirror is used for spectral splitting [19]. Here we propose an alternative that does not need a dichroic mirror, but requires III-V solar cells that are transparent to infrared light. To this end, the epitaxy needs to be lifted off the substrate, a process that in any case is required for sustainability and economical reasons [10]. In our proposal, the silicon solar cells are 
TABLE I

BAND GAPS FOR OPTIMAL YEARLY ENERGY PRODUCTION

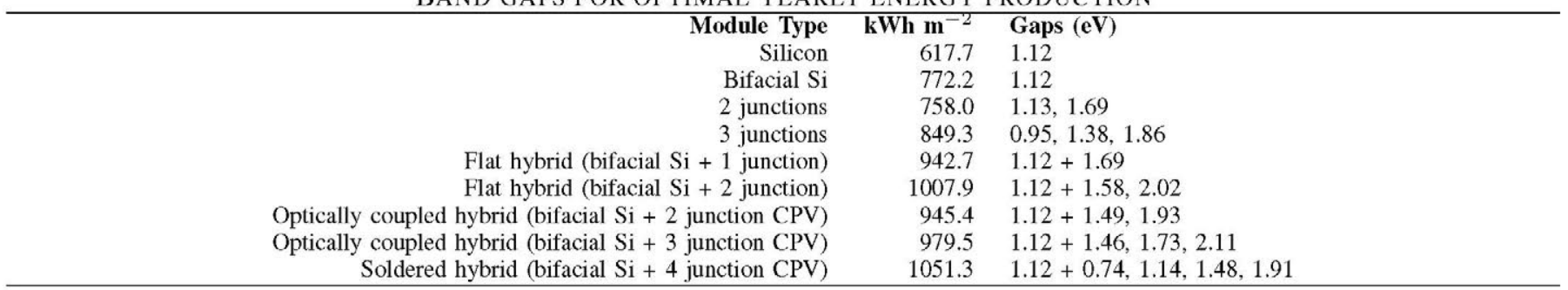

Two axis tracking in Boulder (Colorado) is assumed in all cases. Silicon cells are independently connected. The usable (air mass < 4) yearly global normal irradiation is $2387.9 \mathrm{kWh} \mathrm{m}^{-2}$.

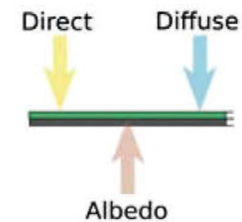

Flat Hybrid 1 junction + Bifacial

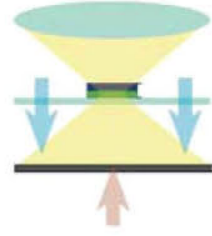

Optical Hybrid 2 junction $+\mathrm{Bi}$.

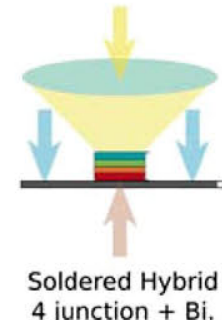

4 junction $+\mathrm{Bi}$
Fig. 11. Schematic description of different types of hybrid modules integrating bifacial silicon with multijunctions. In all cases the silicon solar cells are electrically independent.

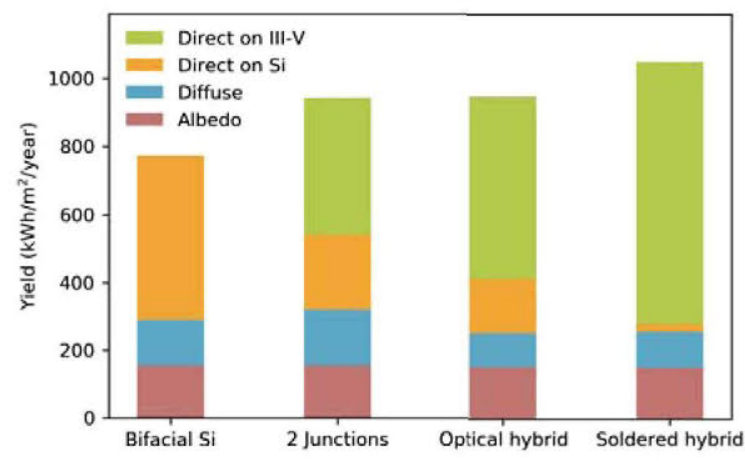

Fig. 12. Energy yield components for bifacial silicon, a 1-sun flat plate dual junction based on bifacial silicon, an optically coupled hybrid (Bifacial Si + 2 junctions), and a soldered hybrid (Bifacial $\mathrm{Si}+4$ junctions).

placed behind the III-V solar cells, preferably away from the focal point of the concentrator optics, as silicon solar cells cannot be used under high concentration, mostly because Auger recombination degrades the voltage. In case of spectral mismatch there is also radiative coupling between the III-V bottom junction and the silicon cell, as the later collects the electroluminescence from the former. This type of module is here called optically coupled hybrid. A schematic description of the different types of hybrid modules here considered is shown in Fig. 11. Optically coupled hybrids might require III-V solar cells limited in size to a fraction of a Watt due to the low thermal conductivity of the glass used as support if no heat sink or other thermal dissipation strategy is used. Small lenses, and consequently small III-V cell sizes might also be required for the soldered hybrid to avoid damaging the silicon solar cells in case of tracker misalignment. With the optically coupled hybrid there is no such risk, as the silicon solar cells are out of the focal plane of the concentrator. In either case, hot-spots or thermal conductivity issues seem to limit the maximum power that can be handled by each multijunction solar cell in these concentrator-bifacial hybrids. This would require recently developed self assembly, microprinting, or massively parallel pick and place techniques to integrate thousands of solar cells per module at low cost [20][25]. Perimeter recombination slightly limits the performance of III-V solar cells smaller than $1 \mathrm{~mm}^{2}$, although this can be countered to some extent with surface passivation [26]. The main advantage of the optically coupled hybrid is that very high energy yields are attainable with dual and triple junctions that should be readily available, as triple junctions have been in commercial production for the past two decades.

Band gaps optimized for maximum yearly energy production are listed in Table I for the hybrid modules in Fig. 11 as well as for conventional flat plate and concentrator multijunctions. As we are interested in determining practical upper limits for photovoltaic performance, here we optimistically assume a spectrally flat $90 \%$ optical efficiency for the concentrator, $80 \%$ transmission of the diffuse irradiance and $5 \%$ of the direct irradiance being scattered by optical imperfections and then collected by the silicon solar cells. The energy yield increase due to back-side albedo irradiance in bifacial cells can reach $50 \%$, but depends on a number of factors [27]. For two axis tracking, low ground cover ratios, panel height larger than the panel dimensions, and a 0.8 albedo, the energy yield increase due to bifacial operation is $25 \%$ [28]. This factor has been multiplied by the energy yield of monofacial flat plate silicon to obtain an optimistic upper limit to the energy yield of bifacial silicon. Other details of our methodology can be found in ref. [5]. The various components contributing to the energy output for each of the hybrid technologies here discussed is presented in Fig. 12

As shown in Table I and Fig. 12, the combination of bifacial and concentrator technology leads to the highest photovoltaic 
energy yields practically attainable due to the harvesting of all components of the irradiance, including the diffuse and back-side albedo contributions. Yearly energy yields near 1 MWh $\mathrm{m}^{-2}$ year $^{-1}$ are attainable in a wide range of locations with any of the three types of hybrid modules here discussed. The soldered hybrid presents the highest yearly energy yields, but optically coupled hybrids might be the ones with the least uncertain path to mass scale production, as the required multijunction cells are less costly, with only marginally lower yearly energy yields.

\section{CONCLUSION}

We have determined the yearly energy yield as a function of multijunction band gaps and other environmental and device parameters. The here described tools should reduce the uncertainties regarding the yearly energy production of multijunctions. These tools can also be used to identify opportunities for cost reduction by tuning those parameters that impact cost but have a relatively small effect on the energy yield. There is a need for solar cell testing standards that better reflect the yearly averaged efficiency, as solar cells designed for record efficiency under the ASTM G173 standard spectra are not optimal for maximum yearly energy yield. We have also considered various types of hybrid modules combining bifacial silicon with multijunctions. We show that energy yields near $1 \mathrm{MWh} \mathrm{m}^{-2}$ year $^{-1}$ are attainable in a wide range of locations with concentrator (Al)GaInP/(Al)GaAs double junctions in combination with bifacial silicon. Further increasing the number of junctions, the energy yield can be increased at most by about $10 \%$.

\section{ACKNOWLEDGMENTS}

Funding was provided by the Goverment of Spain (TEC2015-64189-C3-2-R, ENE2017-91092-EXP, RYC-201415621) and Comunidad de Madrid (P2018/EMT-4308).

\section{REFERENCES}

[1] Fu, R., Feldman, D., Margolis, R., Woodhouse, M. \& Ardani, K. U.S. solar photovoltaic system cost benchmark: Q1 2017. Tech. Rep. NREL/TP-6A20-68925, NREL (2017).

[2] Garcia, I. et al. Spectral binning for energy production calculations and multijunction solar cell design. Progress in Photovoltaics: Research and Applications 26, 48-54 (2018).

[3] Kurtz, S., Myers, D., McMahon, W. E., Geisz, J. \& Steiner, M. A comparison of theoretical efficiencies of multi-junction concentrator solar cells. Progress in Photovoltaics: Research and Applications 16, 537-546 (2008)

[4] McMahon, W. E., Friedman, D. J. \& Geisz, J. F. Multijunction solar cell design revisited: disruption of current matching by atmospheric absorption bands: Disruption of current matching by atmospheric absorption bands. Progress in Photovoltaics: Research and Applications (2017).

[5] Ripalda, J. M., Buencuerpo, J. \& García, I, Solar cell designs by maximizing energy production based on machine learning clustering of spectral variations. Nature Communications 9, 5126 (2018). URL http://www.nature.com/articles/s41467-018-07431-3.

[6] Gueymard, C. A. Prediction and validation of cloudless shortwave solar spectra incident on horizontal, tilted, or tracking surfaces. Solar Energy 82, 260-271 (2008). URL https://linkinghub.elsevier.com/retrieve/pii/ S0038092X07001004.

[7] Sengupta, M. et al. The National Solar Radiation Data Base (NSRDB). Renewable and Sustainable Energy Reviews 89, 51-60 (2018).
[8] Vossier, A., Riverola, A., Chemisana, D., Dollet, A. \& Gueymard, C. A. Is conversion efficiency still relevant to qualify advanced multi-junction solar cells?: Is efficiency relevant with advanced MJ cells? Progress in Photovoltaics: Research and Applications 25, 242-254 (2017).

[9] Essig, S. et al. Raising the one-sun conversion efficiency of III-V/Si solar cells to $32.8 \%$ for two junctions and $35.9 \%$ for three junctions. Nature Energy 2, 17144 (2017)

[10] Horowitz, K. A., Remo, T. W., Smith, B. \& Ptak, A. J. A technoeconomic analysis and cost reduction roadmap for iii-v solar cells. Tech. Rep., NREL (2018).

[11] Green, M. A. et al. Solar cell efficiency tables (Version 53). Progress in Photovoltaics: Research and Applications 27, 3-12 (2019). URL http://doi, wiley.com/10.1002/pip.3102.

[12] Vazquez, M. et al. Reliability of commercial triple junction concentrator solar cells under real climatic conditions and its influence on electricity cost: Reliability of commercial triple junction concentrator solar cells. Progress in Photovoltaics: Research and Applications 25, 905-918 (2017).

[13] Safir, Y. Solar cell module (1995). US Patent Grant 5409550.

[14] Benitez, P., Miñano, J. C. \& Alvarez, R. Photovoltaic concentrator with auxiliary cells collecting diffuse radiation (2008). US Patent Application US20100126556A1.

[15] Martinez, J. F., Steiner, M., Wiesenfarth, M., Glunz, S. W. \& Dimroth, F. Thermal Analysis of Passively Cooled Hybrid CPV Module Using Si Cell as Heat Distributor. IEEE Journal of Photovoltaics 9, 160-166 (2019). URL https://ieeexplore.ieee.org/document/8521666/.

[16] Lee, K.-T. et al. Concentrator photovoltaic module architectures with capabilities for capture and conversion of full global solar radiation. Proceedings of the National Academy of Sciences 113, E8210-E8218 (2016).

[17] Yamada, N. \& Okamoto, K. Experimental measurements of a prototype high concentration Fresnel lens CPV module for the harvesting of diffuse solar radiation. Optics Express 22, A28 (2014). URL https://www.osapublishing.org/oe/abstract.cfm?uri=oe-22-S1-A28.

[18] Yamada, N. \& Hirai, D. Maximization of conversion efficiency based on global normal irradiance using hybrid concentrator photovoltaic architecture: Hybrid concentrator photovoltaic for maximum conversion efficiency, Progress in Photovoltaics: Research and Applications 24, 846-854 (2016). URL http://doi.wiley.com/10.1002/pip.2765.

[19] Mohedano, R. et al. Towards a flat 45\%-efficient concentrator module. AIP Conference Proceedings 1679, 100002 (2015), URL https: //aip.scitation.org/doi/abs/10.1063/1.4931549. https:/aip.scitation.org/ doi/pdf/10.1063/1.4931549.

[20] Mici, J., Ko, J. W., West, J., Jaquith, J. \& Lipson, H. Parallel Electrostatic Grippers for Layered Assembly. Additive Manufacturing (2019). URL https://linkinghub.elsevier.com/retrieve/pii/S2214860419300223.

[21] Sheng, X. et al. Printing-based assembly of quadruple-junction fourterminal microscale solar cells and their use in high-efficiency modules. Nature Materials 13, 593-598 (2014). URL http://www.nature.com/ articles/nmat3946.

[22] Yoon, J. et al. GaAs photovoltaics and optoelectronics using releasable multilayer epitaxial assemblies. Nature 465, 329-333 (2010). URI http://www.nature.com/articles/nature09054.

[23] Srinivasan, U., Liepmann, D. \& Howe, R. T. Microstructure to substrate self-assembly using capillary forces. Journal of Microelectromechanical Systems 10, 17-24 (2001).

[24] Maeda, P. Y. et al. Micro chiplet printer for micro-scale photovoltaic system assembly. In 2015 IEEE 42nd Photovoltaic Specialist Conference (PVSC), 1-6 (2015).

[25] Domínguez, C., Jost, N., Askins, S., Victoria, M. \& Antón, I. A review of the promises and challenges of micro-concentrator photovoltaics, AIP Conference Proceedings 1881, 080003 (2017). URL https://aip. scitation.org/doi/abs/10.1063/1.5001441

[26] Espinet-González, P. et al. Analysis of perimeter recombination in the subcells of gainp/gaas/ge triple-junction solar cells. Progress in Photovoltaics: Research and Applications 23, 874-882 (2015). URL https://onlinelibrary.wiley.com/doi/abs/10.1002/pip.2501.

[27] Luque, A., Lorenzo, E., Sala, G. \& López-Romero, S. Diffusing reflectors for bifacial photovoltaic panels. Solar Cells 13, 277-292 (1985).

[28] Egido, M. A. \& Lorenzo, E. Bifacial Photovoltaic Panels with Sun Tracking. International Journal of Solar Energy 4, 97-107 (1986). 